\title{
Dynamic Guidance by Colored Running Lights and Af- fordance: Route Choices of Adults and Older Children
}

\author{
Laura Künzer, Robert Zinke, Gesine Hofinger \\ Team HF Human Factors Research and Training \\ Hofinger, Künzer \& Mähler PartG \\ Ludwigsburg, Germany \\ laura.kuenzer@team-hf.de; robert.zinke@team-hf.de; gesine.hofinger@team-hf.de
}

\begin{abstract}
Guidance to emergency exits play an important role for safe evacuation. Dynamic route guidance by colored flashing lights and strobe lights at emergency exits has been tested [1-3], but the effects of dynamic lights to support route choices need to be determined in more detail. Also, the guidance effects of different colors need to be examined and the reaction of various groups of evacuees.

The paper analyzes the effects of red and green running lights on route choice in subway stations comparing adults and older children (10 to 12 years old). Data was gathered in a laboratory experiment, focusing on the concept of affordance $[4,5]$. Participants were asked to make a decision about the safest direction between two alternative directions. Their choice was either unsupported or supported by red or green running lights.

In general, an interaction between color and direction of the running light was found. Green running lights influenced route choices of both participant groups and led participants clearly into the direction indicated by the lights. Red running lights influenced route choices of both participant groups, but red lights lead to ambiguous decisions. Architectural elements such as stairs influenced route choices of both participant groups (functional affordance). But green running light offered a stronger indication to a safe route (cognitive affordance) than a visible staircase (functional affordance). Green lights even led participants to modify their route preference. In contrast, red running lights had an aversive effect: older children chose against the lights and preferred the other direction than the red lights were directing to. Implications for design of dynamic route guidance are discussed. This includes colored running lights to lead evacuees to a safe exit and to implement the influence of running lights on route choice and movement in simulations.
\end{abstract}

Keywords: evacuation, guidance, red and green lights, route choice, adults, older children, emergency exit

\section{Motivation of the study and the concept of affordance}

In case of fire, guidance to the safest emergency exits plays an important role in evacuations. In order to support wayfinding and route choice of evacuees, the design of emergency routes has to fulfill requirements. Requirements defined in fire regulation codes include number of exits, length and special dimension of escape routes as well as emergency lighting. Yet, additional design aspects and features could be considered in order to foster wayfinding and quick decision making.

In this regard, the possibility of dynamic route guidance by colored flashing lights and strobe lights at emergency exits has been tested [1,2]. For example, it is known that "running lights" can create a sense of direction [6]. But the effects of dynamic lights to support route choices still need to be determined in more detail. Also, the effect of different colors needs to be examined in more detail as well as the reaction of various groups of evacuees.

This study focuses on the colors green and red because both colors are used to indicate emergency exits. Results of previous studies indicate meanings of colors: green to indicates "safe" or "go"; red represents "stop" or "danger" [8]. Depending on the country and infrastructure, the use of color for guidance in the context of danger varies to some extent. While the green pictogram of a "running man" indicates a safe 
emergency route or exit in most European and Asian countries e.g. [7], the red "EXIT"-sign is used for the same purpose in parts of the USA.

In this paper, the effects of red and green running lights on route choice were analyzed comparing adults and older children from Germany, the latter ranging from 10 to 12 years in age. Older children were chosen to participate because in some countries children at this age use public transport on their own and therefore will also need to understand dynamical guidance features. Younger children are expected to be accompanied by parents or other adults.

The comparison of data from both participant groups focuses on the theoretical concept of affordance [4]. "Affordance" has different notions. In the user-centered design view by Norman [9], affordances result from a mental representation based on knowledge and past experiences. This means that specific characteristics of an object are related to specific actions and knowledge of a user. For example, a person may have encountered "stairs" before and may have made the experience that using them leads upstairs or downstairs. Three kinds of affordances are differentiated: sensory, cognitive and functional affordance $[5,10]$. Within this study all three kinds of affordances are taken into consideration:

- "Sensory affordance" helps users with sensory actions (e.g. seeing, hearing, or feeling) and therefore detecting information. Running lights can attract attention. In this study, sensory affordance of green and red running lights was tested with route choices against a baseline without running lights.

- "Cognitive affordance" as a design feature helps interpreting the intended function of a design element. With respect to cognitive affordance, one objective of the study is to determine if a green running light indicates movement into a specific direction.

- "Functional affordance" combines both sensory and cognitive affordance. It allows to identify how and for what aim (usage and utility) an object can be used. The functional affordance of stairs was assumed to be "upstairs" or "outside" in this study.

Affordance in general depends on knowledge and experience of the individual. Since experience also varies with age, results were compared among children and adult participants.

\section{Method}

\subsection{Item design}

Pictures of five intersections were taken in subway stations in Berlin and were digitally edited for the purpose of the study. The intersections were presented on a laptop computer as motion pictures in graphics interchange format (GIF). Dynamic guidance was presented in the GIFs by green and red running lights, pointing into a certain direction, e.g. starting from the left and moving to the right. The running lights were moving with a frequency of $200 \mathrm{~ms}$ either to the left or to the right.

Each item was designed without any lights (baseline; examples fig. 1-3), and with either green or red running lights (examples fig. 4 and 5). In total, 25 different item designs were used (table 1). 
Table 1: Items designs for intersections 1 to 5 , no lights (baseline), color of light and direction of running lights, 25 items in total.

\begin{tabular}{|c|c|c|c|}
\hline Intersection & No lights & Color of lights & Direction of running light \\
\hline \multirow{2}{*}{ Intersection 1} & \multirow{2}{*}{ Baseline } & Green & $\begin{array}{l}\text { Left } \underset{\leftarrow}{\leftarrow} \\
\text { Right } \rightarrow\end{array}$ \\
\hline & & Red & $\begin{array}{l}\text { Left } \underset{ }{\leftarrow} \\
\text { Right } \rightarrow\end{array}$ \\
\hline \multirow{2}{*}{ Intersection 2} & \multirow{2}{*}{ Baseline } & Green & $\begin{array}{ll}\text { Left / upstairs } & \mathbf{K} \\
\text { Right / along platform } & \rightarrow\end{array}$ \\
\hline & & Red & $\begin{array}{ll}\text { Left / upstairs } & \mathbf{K} \\
\text { Right / along platform } & \rightarrow\end{array}$ \\
\hline \multirow{2}{*}{ Intersection 3} & \multirow{2}{*}{ Baseline } & Green & $\begin{array}{c}\text { Left } \underset{\mathbf{r}}{\mathbf{\leftrightarrow}} \\
\text { Right / upstairs } \rightarrow\end{array}$ \\
\hline & & Red & 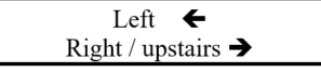 \\
\hline \multirow{2}{*}{ Intersection 4} & \multirow{2}{*}{ Baseline } & Green & $\begin{array}{l}\text { Left } \underset{\leftarrow}{\leftarrow} \\
\text { Right } \rightarrow\end{array}$ \\
\hline & & Red & $\begin{array}{l}\text { Left } \underset{ }{\leftarrow} \\
\text { Right } \rightarrow\end{array}$ \\
\hline \multirow{2}{*}{ Intersection 5} & \multirow{2}{*}{ Baseline } & Green & $\begin{array}{l}\text { Left } \underset{\leftarrow}{\leftarrow} \\
\text { Right } \rightarrow\end{array}$ \\
\hline & & Red & $\begin{array}{l}\text { Left } \underset{ }{\leftarrow} \\
\text { Right } \rightarrow\end{array}$ \\
\hline Sum of Items & 5 & & 20 \\
\hline
\end{tabular}

The design of the presented intersections was "equivalent" for intersections 1, 4 and 5 (figures 1-3). These intersection, when presented without running lights (baseline), offered equivalent route options for the symmetric left and right directions. Equivalent means that both routes had the similar length and there were no stairs on the route to the exit. Also there was no signage in either direction. So, they were equivalent considering the fastest route to a safe exit. On the other hand, for intersection 4 and 5, the participants' position was assumed to be on a staircase or escalator, so one of the routes was a bit shorter.

The designs of the remaining intersections 2 and 3 were "not equivalent" as the choice was between a near staircase and route along the platform (intersection 2, see fig.6) or between stairs and a longer route to the left that also led to another, hardly visible, staircase (intersection 3, see fig. 7).

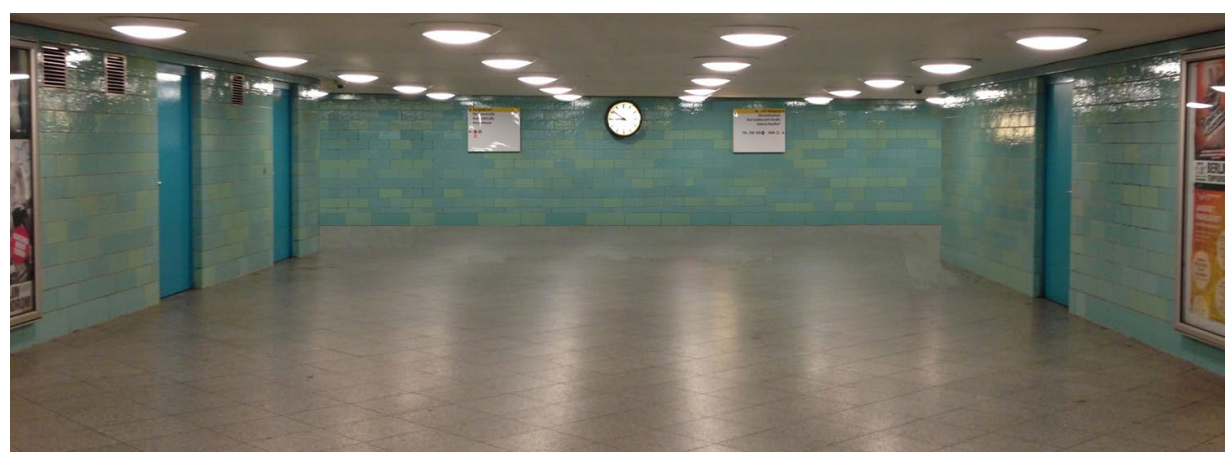

Fig. 1: Intersection 1 without lights (baseline) and equivalent route options for symmetric left and right direction.

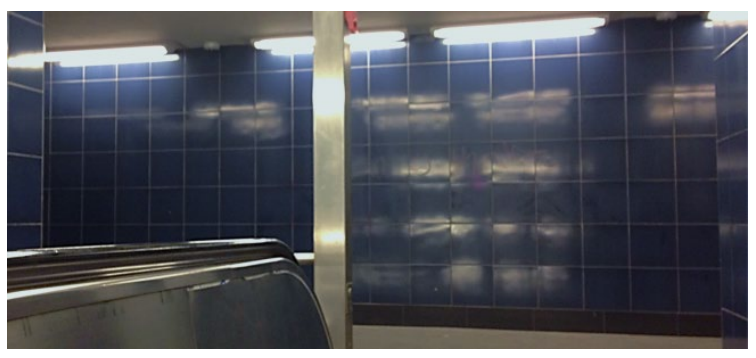

Fig. 2: Intersection 4 without lights (baseline) and equivalent route options for symmetric left and right direction but with a shorter route on the right side.

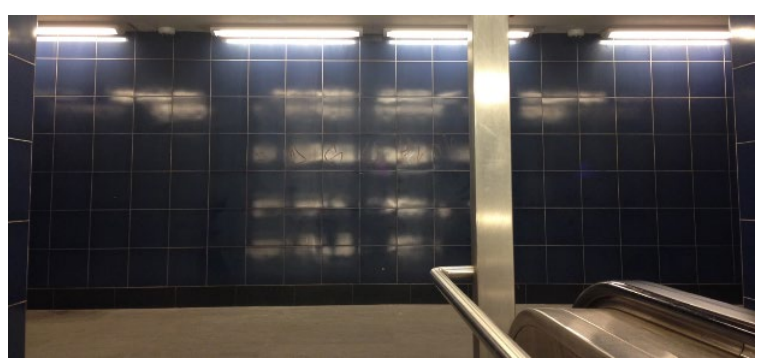

Fig. 3: Intersection 5 without lights (baseline) and equivalent route options for symmetric left and right direction but with a shorter route on the left side. 


\subsection{Participants}

50 participants took part in the study, 31 adults and 19 older children. The age of the adults ranged from 20 to 31 years with an average of $24.87 ; \mathrm{SD}=2.86$. The age of the children ranged from 10 to 12 years with an average of $10.79 ; \mathrm{SD}=.63$.

The study was conducted in German. All adults were Germans, as were 14 of the older children, the others five older children stated to be from Bulgaria (2), Romania (1) and Turkey (2). All older children confirmed that they were able to easily understand and follow the German instructions of the study. Only two of the participants were left-handed. Due to this small number, a possible effect of handedness was not further examined. 25 of adult participants were bachelor or master students. No participant had a professional background related to colored light or signals, so effects of professional background were also not further examined. All participants stated to have normal or corrected eye vision.

\subsection{Procedure of the study}

All participants were informed about the procedure and risks of the study. Additional information about the research project ORPHEUS was provided. Then informed consent was obtained from all participants. Informed consent for the older children were signed by their parents. The study with the older children was conducted at a school in the presence of the children's parents. The study with the adults was conducted in a real subway station accompanying another field study [12].

Participants filled a questionnaire about socio-demographic data, e.g. age, nationality, eye vision including their experience with subway systems. The participants were then seated in front of a laptop computer. The items and additional questions were presented in a digital format.

Participants were instructed to imagine that they were in a subway station when the station had to be evacuated due to a fire alarm. They were told that several pictures showing two alternative exits are to be presented in sequence and that their task for every picture was to choose the safer of the two exits. Their choice, e.g. for intersection 2 to turn right or to go upstairs (see fig. 6) was either unsupported (baseline) or supported by red or green running lights.

Participants stated their decision via keyboard. The five items without any lights were presented first in randomized order as a baseline. Then the 20 items with green or red running lights were presented in a randomized order. Finally, the participants answered a questionnaire about the green and red lights.

\section{Research questions and results}

The study included the following research questions:

1. Is there a preference of direction when no running lights influence route choice?

2. How are route choices influenced by green or red running lights?

3. Is there an effect of functional affordance by architectural design on route choice?

4. Is the effect of running light (sensory affordance) stronger or weaker than the functional affordance of architectural design?

5. Are there any differences between the choices of adults and older children?

Items of intersections 1, 4 and 5 (equivalent options) were included for research question number 1 and 2. For question 3 and 4, the items of intersections 2 and 3 were included.

To allow for comparisons (research question number 5), all items were statistically analyzed separately for adult and older children. The comparisons will be discussed as supplementary part of research questions 1 to 4 .

\subsection{Is there a preference of direction when no running lights influence route choice?}

As most participants were right-handed and Germans, there was the expectation that there would be a preference for the right side when there is no guidance by signage or light and when route options have an architectural equivalence. This kind of "natural" preference of direction was analyzed by the items of the baseline condition without any running lights (equivalent intersections 1, 4 and 5). Tests of binomial-distribution were used to analyze "natural" preferences. 
No "natural" preference for the left or right direction was found for participants' choices in intersection 1 (see fig. 1) and intersection 4 (see fig. 2). Also, no "natural" preference was found in older children's choices for intersection 5 (see fig. 3). Thus, data supported the assumption of equivalent intersection design. Also, the right-handedness and nationality of most of the participants do not influence their route choice in the condition of architectural equivalence.

A "natural" preference to turn right (25 out of 31) was only found in the data of the adults for intersection 5 (see fig. 3). Intersection 5 has symmetric route options, and at the same time has a possible slight affordance for the left side due to the escalator on the right sight impeding view, as stated above. Only for this item in the baseline condition the assumed "natural" preference was found. This is surprising because in this item there was even slight functional affordance suggesting the left hand option. Why this is the case remains unclear from the data available.

\subsection{How are route choices influenced by green or red running lights?}

The effect of lights was analyzed by data comparison of the baseline and participants' route choices with green or red running lights. McNemar tests were used to analyze "changes" in route choices when lights were included in the equivalent intersections 1,4 and 5.

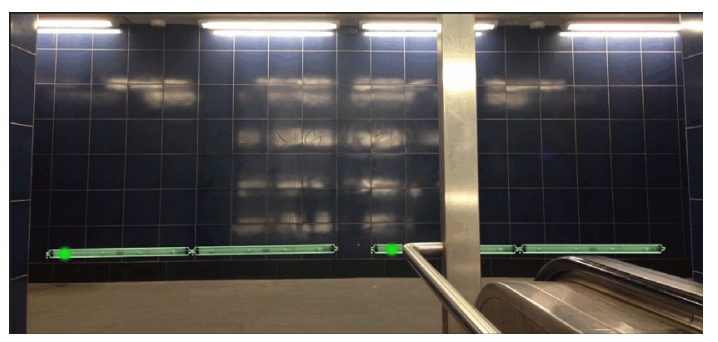

Fig. 4: Intersection 5 with green running lights moving to the left (movement was achieved by using GIF).

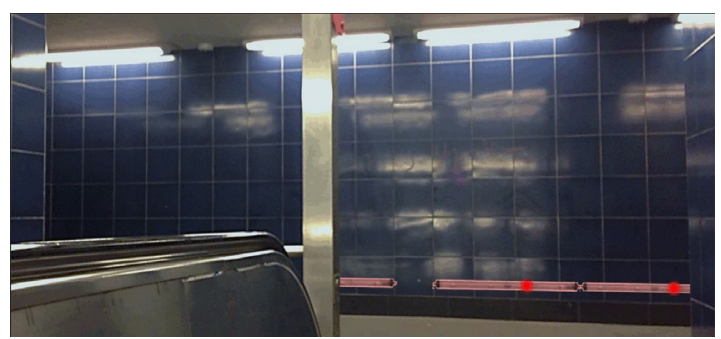

Fig. 5: Intersection 4 with red running lights moving to the right (movement was achieved by using GIF).

Green running lights influence route choices of both participant groups leading participants into the direction the light is moving to.

For intersection 1 and 4, where no "natural" preference was found in the baseline condition, both participant groups' route choices changed significantly to the direction the green running lights were moving to. This effect was found to be independent of the direction of the green running light: Most participants distinctly chose the direction of the running lights, no matter if the green light moved to the left or to the right. The same effect was found for intersection 5 for most older children's choices. For intersection 5 (see fig. 4), where a "natural" preference "to the right" was found for adults, most adults also significantly preferred to follow the green lights, even when the lights were moving to the left.

\section{Red running lights influence route choices of both participant groups, but effect of red lights is differ- ent for adults and older children}

For intersection 1 (equivalent routes), most participants in both groups chose not to follow the direction of the red running light. Instead, they preferred the other route possibility, e.g. most participants chose to turn to the left if the red running light pointed to the right.

Results for intersections 4 and 5 (see example in fig. 5) showed differences between both participant groups: around $60 \%$ of the adults chose the direction the red lights were moving to. Also around $60 \%$ of the older children chose not to follow the directions of the red running lights and instead opted for the other direction.

In summary, running lights clearly influence route choice compared to the baseline. Comparing results for red and green lights, it can be stated, that participants' choices to follow the green lights were found to be significant more often (compared to the baseline and the red running lights). Results were also clearer than choices to follow the red running lights. Some adults chose to follow red lights which could be explained by sensory affordance: "movement of lights leads to a safe exit" regardless of the color of the 
running light. For older children, the red running light led to a decision against the direction the red light was moving to. The color red represents "stop" as in traffic lights or "danger" [8]. Older children might have projected this concept from their knowledge to the red running lights (cognitive affordance). So, it can be concluded that the conflict between the cognitive affordance of the color red and the sensory and cognitive affordance of the movement of the light into a certain direction overall leads to a less definite, more ambiguous decisions.

\subsection{Is there an effect of functional affordance by architectural design on route choice?}

Intersections 2 and 3 without any running lights (baseline) were used to analyze the influence of functional affordance offered by "architectural elements". Examples can be found in figures 6 and 7 that show intersections 2 and 3 (note that both intersections were presented in different items with all variations of green and red running lights moving to the left or to the right).

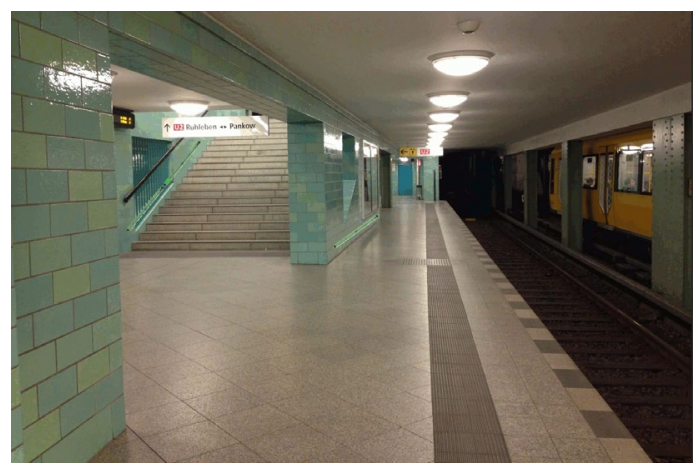

Fig. 6: Intersection 2 with green running lights moving to the left/ upstairs.

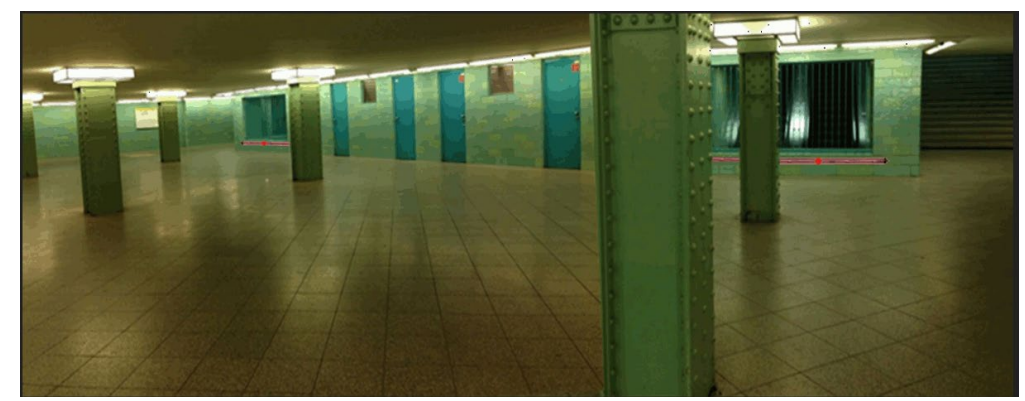

Fig. 7: Intersection 3 with red running lights moving to the right/ upstairs.

\section{"Stairs" influence route choices of both participant groups but in different ways.}

Overall, the architectural element "visible stairs" was statistically more often chosen as safest route. This was in accordance with expectations, as the staircase in both items is clearly visible and nearer than the other exit. Also, a staircase might be known to lead "up" or "outside" the subway station thereby indicating "a safe route" (functional affordance).

Differences are found between the groups: Older children chose significantly more often to walk upstairs in intersection 2 whereas no significant effect was found for the adults. One possible explanation is that for adults climbing up stairs is more exhausting and therefore some of them chose the longer route without stairs.

On the other hand, adults chose significantly more often to walk upstairs in intersection 3 whereas no significant effect was found for the older children. Here, one possible explanation is that the children didn't recognize the staircase that was hardly visibly on the left.

Despite the effects we found, data of this study do not allow generalized interpretations to how staircases and perceived distances influence route choices. 


\subsection{Is the effect of running light (sensory affordance) stronger or weaker than the func- tional affordance of architectural design?}

\section{The green running light had a stronger influence on participants' choices (cognitive affordance) than the visible staircase (functional affordance) and even led participants to modify their route preference.}

By comparing the baseline (no running lights) of intersections 2 or 3 with items including running lights (green or red), interaction effects of cognitive affordance and functional affordance were analyzed using binomial tests. Examples of items with colored running lights can be found in figures 6 and 7 .

For intersections 2 and 3, most adults choose the direction the running lights were moving to. Detailed analysis showed that green lights had a significant effect on route choice. The cognitive affordance of the green lights led to a more definite route choice than the functional affordance of the stairs. If the green running light moved "upstairs", the positive effect by green lights increased and even more participants decided to walk upstairs. The effect, redundancy gain [11], can be explained by congruence of the cognitive affordance of the green light and the functional affordance of the viewable staircase.

Another statistically significant effect was found for intersection 2 in the condition of green lights directing "along the platform". Around $84 \%$ of the adults chose to follow the direction of the running green lights. This is change in route choice compared to the baseline (where they mostly "walked" upstairs) was caused by the sensory and cognitive affordance of the green light. This same effect was found for intersection 3 where adults even chose to walk to the left instead of going directly upstairs (to the right) which had been their choice in the baseline condition. Thus, sensory and cognitive affordance of the green running lights seems to be stronger than the functional affordance of the stairs.

Results from older children also show a strong significant effect of green running lights guiding to the direction of movement of the lights. With green running lights moving in the upstairs direction, most older children still chose to walk upstairs. But when the green running lights were moving along the platform, older children also chose the route along the platform in intersection 2 - which meant a change of route choice in favor of the direction of the green moving lights. This same effect was found for the adults.

Red lights lead to a greater variability of route choices. Interaction (or better interference) between cognitive and functional affordance and the red running light was found. For intersection 2, with a red light moving upstairs, participants more often chose to walk along the platform. When the red light moved along the platform, adults still chose to walk along the platform. Although some adults chose to follow the red running lights, some changed their route choice against the direction the red running lights moved to. With respect to following or avoiding the direction of movement of the red running lights, no statistical evidence was found for either one of the two route choice preferences. In contrast, for the older children red running lights had an aversive effect: they avoided the direction of movement of the red lights and preferred the other route available.

Summarizing results, it can be said that the effect of red running light in comparison to distances or stairs cannot easily to determined. On the other hand, for both participant groups, cognitive affordance of the green running lights seems to be stronger than the functional affordance of the stairs.

\section{Limitations of the study and further research}

Only five different, but modified intersections were included. Also the intersections were only shown in an experimental setting on the laptop computer. Future studies should be carried out in architectural settings, e.g. subway stations using actual guiding lights. Also, the effects of different saturation or lightness of the colors green and red and combinations of colors and pictographs, e.g. green arrows; red crosses, should be determined.

The study included only two participant groups. To establish a generalization of the findings, further research will include different participant groups, e.g. elderly, differences in gender and cultures, and groups with special conditions, e.g. people with color-blindness. Also, a greater number of participants for each group should be included, because the sample discussed here allowed only non-parametrical testing. Some effects should be investigated based upon a greater sample, e.g. a preference for a direction of people from countries with left-hand traffic, or possible effects of handedness of participants. To examine 
redundancy gain effects and to include more participant groups, e.g. people with impaired vision, sensory affordance by guiding lights should be combined with guiding sounds.

\section{Conclusions}

In the study presented, strong effects of running lights and several types of interactions between color, direction if the light and architectural elements were found. Green running lights influence route choices of both, adult participants and older children, and lead into the direction of movement of the green lights. Green running lights were even stronger than functional affordance proposed by the design of architectural elements in the intersections. Conclusions for evacuation guidance and simulations are as follows:

- Installation of running lights at emergency routes is advisable and can lead evacuees to a safe exit.

- Variability in the interpretations of the color red indicates that dynamic guidance with running lights should use green lights. The exact color values of green are yet to be determined.

- Furthermore, the influence of running lights on route choice and movement should be implemented in simulations: modelling pedestrian flows for the evacuation from an infrastructure could gain an increased level of realism with respect to evacuees' route choice by including route choice preferences in favor of the movement of green running lights. This in return could make evacuation simulation more effective for predicting flows and required capacities of escape routes when using dynamic route guidance by green running lights.

\section{Acknowledgements}

This research was funded by the German Ministry for Education and Research (BMBF) contract No. 13N13266 (project ORPHEUS). The authors also would like to thank all participants of the study, especially the children and their parents. Prof. Dr. Andreas Pflitsch and his team encouraged their students to take part in the experiment. Anna-Lisa Mews and Lisbeth Balters conducted the children's' study.

\section{References}

[1] D. Nilsson, H. Frantzich, and W. Saunders, "Coloured Flashing Lights To Mark Emergency Exits - Experiences From Evacuation Experiments," (en), Fire Safety Science, vol. 8, pp. 569-579, 2005.

[2] L. Künzer, G. Hofinger, and R. Zinke, "The Influence of Colored Running Lights on Route Choice Dynamic Guidance and Affordance," in Proceedings of the $8^{\text {th }}$ International Conference on Pedestrian and Evacuation Dynamics: PED 2016, Hefei, 2016, pp. 167-172.

[3] E. R. Galea, D. Cooney, and L. Filippidis, "Active dynamic signage system: A full-sclale evacuation trial," Human Behaviour in Fire, 303-314,, 2015.

[4] J. J. Gibson, The ecological approach to visual perception. Boston: Houghton Mifflin, 1979.

[5] R. Hartson, "Cognitive, physical, sensory, and functional affordances in interaction design," Behaviour \& Information Technology, vol. 22, no. 5, pp. 315-338, 2003.

[6] E. B. Goldstein, Wahrnehmungspsychologie, 2nd ed. Heidelberg: Spektrum, 2002.

[7] Bundesanstalt für Arbeitsschutz und Arbeitsmedizin, "Technische Regeln für Arbeitsstätten ASR A2.3: Fluchtwege, Notausgänge, Flucht- und Rettungsplan“ (Technical workplace regulations for escape routes, escape exits and escape route plans). GMB1. Nr.45 vom September 2007, die zuletzt im Januar 2017 geändert wurde," 2007.

[8] L. Künzer, ““'Alarmstufe Rot!“ oder „Alles im grünen Bereich!“: Farben im Kontext von Gefahr und Sicherheit," ("Red Alert" or "Green is Safe" Colors in the Context of Danger and Safety), Dissertation, Universität Regensburg, Regensburg, 2016.

[9] D. A. Norman, "Affordances: Commentary on the Special Issue of AI EDAM," AIEDAM, vol. 29, no. 03, pp. 235-238, 2015.

[10] E. Carattin, R. Lovreglio, E. Ronchi, and D. Nilsson, "Affordance-Based Evaluation of Signage Design for Areas of Refuge," Interflam 2016. Fire Science and Engineering Conference, pp. 781-786, 2016.

[11] C. D. Wickens and J. G. Hollands, Engineering psychology and human performance, $3^{\text {rd }}$ ed. Upper Saddle River, NJ: Prentice Hall, 2000. 